Incident air kerma to absorbed organ dose conversion factors for breast and lung in PA thorax radiography : The effect of patient thickness and radiation quality

Kelaranta, A.

2016-12

Kelaranta , A , Toroi , P \& Vock , P 2016 , ' Incident air kerma to absorbed organ dose conversion factors for breast and lung in PA thorax radiography : The effect of patient thickness and radiation quality ' , Physica Medica , vol. 32 , no. 12 , pp. 1594-1601 . https://doi.org/10.1016/j.ejmp.20

http://hdl.handle.net/10138/232107

https://doi.org/10.1016/j.ejmp.2016.11.113

publishedVersion

Downloaded from Helda, University of Helsinki institutional repository.

This is an electronic reprint of the original article.

This reprint may differ from the original in pagination and typographic detail.

Please cite the original version. 
Original paper

\title{
Incident air kerma to absorbed organ dose conversion factors for breast and lung in PA thorax radiography: The effect of patient thickness and radiation quality
}

\author{
A. Kelaranta ${ }^{\mathrm{a}, \mathrm{b}, \mathrm{c}, *}$, P. Toroi ${ }^{\mathrm{a}, \mathrm{d}}$, P. Vock $^{\mathrm{d}}$

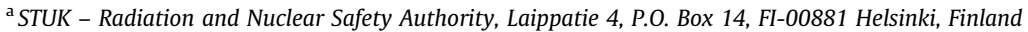 \\ ${ }^{\mathrm{b}}$ HUS Medical Imaging Center, University of Helsinki and Helsinki University Hospital, PO Box 340, FI-00290 Helsinki, Finland \\ ${ }^{c}$ Department of Physics, University of Helsinki, P.O. Box 64, Helsinki FI-00014, Finland \\ ${ }^{\mathrm{d}}$ Inselspital University Hospital, Freiburgstrasse 10, CH-3010 Bern, Switzerland
}

\section{A R T I C L E I N F O}

\section{Article history:}

Received 21 April 2016

Received in Revised form 3 November 2016

Accepted 21 November 2016

Available online 30 November 2016

\section{Keywords:}

Organ dose

Conversion factor

Thorax X-ray examination

Radiation quality

Patient thickness

\begin{abstract}
A B S T R A C T
Purpose: Converting the measurable quantities to patient organ doses in projection radiography is usually based on a standard-sized patient model and a specific radiation quality, which are likely to differ from the real situation. Large inaccuracies can therefore be obtained in organ doses, because organ doses are dependent on the exposure parameters, exposure geometry and patient anatomy. In this study, the effect of radiation quality and patient thickness on the organ dose conversion factors were determined. Methods: In this study, the posterior-anterior projection radiograph of the thorax was selected in order to determine the effect of radiation quality (tube voltages of $70-130 \mathrm{kV}$ and total filtrations of $3 \mathrm{mmAl}$ to $4 \mathrm{mmAl}+0.2 \mathrm{mmCu}$ ) and patient thickness (anterior-posterior thicknesses of $19.4-30.8 \mathrm{~cm}$ ) on the breast and lung dose conversion factors. For this purpose, Monte Carlo simulation programs ImpactMC and PCXMC were used with computed tomography examination data of adult male and female patients and mathematical hermaphrodite phantoms, respectively.

Results: Compared to the reference beam quality and patient thickness, the relative variation range in organ dose conversion factors was up to $74 \%$ for different radiation qualities and $122 \%$ for different patient thicknesses.

Conclusions: Conversion factors should only be used with comprehensive understanding of the exposure conditions, considering the exposure parameters, exposure geometry and patient anatomy they are valid for. This study demonstrates that patient thickness-specific and radiation quality-specific conversion factors are needed in projection radiography.
\end{abstract}

๔ 2016 Associazione Italiana di Fisica Medica. Published by Elsevier Ltd. All rights reserved.

\section{Introduction}

According to current knowledge, the risk of radiation-induced cancer is assumed to be linearly dependent on the cumulated radiation dose [1]. The International Commission on Radiological Protection (ICRP) does not recommend the effective dose to be used for individual risk assessment [1]. The absorbed organ dose is a better measure for estimating the patient risk than the effective dose $[1-3]$. Estimating the radiation-induced risk of cancer to the patient from an X-ray examination requires knowledge of organ doses, not just the dosimetric quantities used for dose monitoring.

\footnotetext{
* Corresponding author at: HUS Medical Imaging Center, University of Helsinki and Helsinki University Hospital, PO Box 340, FI-00290 Helsinki, Finland.

E-mail address: anna.kelaranta@alumni.helsinki.fi (A. Kelaranta).
}

Accurate estimation of the radiation dose to the patient can be based on conversion factors from measured dose data to patient organ doses by using information about the exposure parameters, exposure geometry and patient anatomy. The patient models used for calculating conversion factors are typically standard-sized and do not consider differences in patient anatomy.

A report by the American Association of Physicists in Medicine (AAPM) provides guidance for patient size corrections in computed tomography (CT) imaging [4], and can therefore be used in estimating patient size-specific doses from measured dose data (in terms of $\mathrm{CT}$ air kerma index, $\mathrm{C}_{\mathrm{K}}$ ). $\mathrm{C}_{\mathrm{K}}$ is already closer to organ doses than the measurable quantities in projection imaging because it is measured in a phantom, and the internal phantom and patient dose distributions are more uniform in CT than in projection imaging. In projection imaging, the dose to an organ is high on the entrance side of the $\mathrm{X}$-ray beam and low on the exit side. Therefore, much larger 
variations in organ doses can be expected in projection imaging than in CT. The dose levels in interventional radiology are many orders of magnitude higher than in conventional projection imaging, and sometimes even higher than in CT. However, there are currently no generally used correction factors for patient size in conventional projection imaging and interventional radiology. The best accuracy in patient dose estimation can be achieved when individual and examination-specific simulation is performed based on CT data of the patient. This method is commonly used in radiation therapy treatment planning. However, this method is very time-consuming and, due to the lack of appropriate CT data, not practical on a routine basis for dose estimation in X-ray imaging.

Different levels of accuracy are needed for organ dose estimations depending on the purpose of use. Some publications include tabulated organ and effective doses for certain diagnostic procedures and examinations $[5,6]$. Typical dose values are usually accurate enough as estimates for the order of magnitude of the exposure and subsequent cancer risk. However, the use of such values is not sufficient for accurate dose calculations for individual patients due to the individual patient and examination conditions. If a tabulated value alone is used for patient dose estimation, the unknown incident dose level adds additional uncertainty to the estimation and may have a major effect on individual doses. Therefore, more accurate dose conversion factors from measured values to organ doses are needed for research and the optimization of patient doses. Conversion factors are typically given for a specific examination type and for a single or a few radiation qualities with certain assumptions about the beam size, its positioning, and the patient size [5-9] and determined by anthropomorphic phantom measurements or Monte Carlo simulations. However, differences in patient size are rarely taken into account. There are various software programs designed to calculate organ doses in diagnostic X-ray examinations, for example XDOSE [10], CALDose_X [11], PCXMC [12] and ImpactMC $[13,14]$. According to the results of a Coordinated Research Project (CRP E2.10.08) of the International Atomic Energy Agency [15], the most common methods for effective and organ dose estimation in projection imaging were PCXMC [12] and NRPB-R262 and NRPB-SR262 [6,16].

Posterior-anterior (PA) examination of the thorax is one of the most common radiographic examinations in projection X-ray imaging [17-19]. In thorax PA examination, special attention should be paid to the two most radio-sensitive chest organs, the breasts and the lungs, which are located almost entirely within the primary radiation beam. The tissue weighting factor for the breast has been increased from 0.05 to 0.12 when comparing ICRP Publications 60 and $103[1,20]$, and has reached the value of the lung. This increase is due to the evidence on the radiosensitivity of the breast and because more emphasis has been put on cancer incidence rather than cancer mortality by the ICRP.

The aim of this study was to determine the effect of patient thickness on the dose conversion factors by Monte Carlo simulations of a thorax PA examination, based on CT examination data of adult male and female patients with different anterior-posterior thicknesses and thickness-adjusted mathematical hermaphrodite phantoms. Moreover, the effect of different radiation qualities based on a standard-sized mathematical hermaphrodite phantom was determined.

\section{Materials and methods}

Risk estimates are normally based on organ doses or the effective dose, which cannot be directly measured. Therefore, measurable quantities based on air kerma are generally used for clinical dosimetry. In the energy range used in X-ray diagnostics, air kerma equals the dose absorbed to air, and air kerma is therefore the quantity used throughout this paper, as it is internationally recommended $[21,22]$.

ImpactMC (version 1.4.0.0, 2014) from CT Imaging, Erlangen, Germany [13,14], allows the user to generate 3D dose distributions with user-defined acquisition parameters from retrospective volume CT data. ImpactMC was designed to simulate CT devices but it can also be used to simulate projection radiography [23]. There are no validation articles for the use of ImpactMC for projection radiography so far, but the localizer mode can be used for this purpose in ImpactMC with adapted geometry and spectra (Paul Deak, November 1, 2016, personal communication) [24,25]. In this study, ImpactMC was used to determine the effect of patient thickness on conversion factors. Anonymised CT patient data from 5 male and 5 female adult patients investigated in 2011 at the Inselspital University Hospital, Bern, Switzerland, were used in ImpactMC simulations. The patient selection criteria were that (a) the patients had undergone trauma $\mathrm{CT}$, (b) the scanned region covered the lungs and (c) both genders were represented equally by 5 five different patient sizes, ranging from extra small (XS) to small (S), medium (M), large (L), and extra large (XL) (see Table 1). The patient thickness measured at the mid-sagittal plane at the mamilla level in the anterior-posterior (AP) dimension (see Fig. 1) was used to define the patient size. The patients used in ImpactMC simulations had been scanned in a supine position with the trauma CT protocol at a slice thickness of $1.5 \mathrm{~mm}$, and the Siemens convolution kernel was B20f. The reference X-ray spectrum (X-ray tube voltage, anode angle, filtration, see Table 2) used in ImpactMC simulations was generated using Spektripaja 3.0 program [26]. The Be window used in Spektripaja was $0.8 \mathrm{~mm}$, and the air layer thickness was $1 \mathrm{~m}$.

Prior to the ImpactMC simulations, the patient table was removed from the DICOM images by thresholding and masking in the computer programs 3DSlicer (version 4.4) and Matlab (version $\mathrm{R} 2014 \mathrm{~b}$ ). The position of the X-ray detector was estimated to be at the sternum position in order to fix the position similarly for male and female patients. The X-ray beam size was adjusted to $900 \mathrm{~cm}^{2}$ $(30 \mathrm{~cm} \times 30 \mathrm{~cm})$ so that it covered the lungs of all patients at the same focus to detector distance (FDD) of $200 \mathrm{~cm}$ (see Fig. 2). The default density and material definition files in ImpactMC were used. An air kerma output (input parameter in ImpactMC) of $1 \mathrm{mGy}$ was assumed at the FDD and the incident air kerma at the focus to skin distance (FSD) was then calculated based on the patient thickness. Organ dose conversion factors were calculated relative to the incident air kerma. If conversion factors for the air kerma-area product are needed, these values are divided by the field size at the patient entrance surface. If conversion factors from the entrance surface air kerma are needed, these values are divided by the backscatter factor, which is close to 1.4 [27] in this case.

Table 1

Patient thickness was measured at the patient?s midsagittal plane at the mamilla level in the anterior?posterior (AP) dimension.

\begin{tabular}{ll}
\hline Patient size $^{\mathrm{a}}$ & AP thickness $(\mathrm{cm})$ \\
\hline MXS & 19.4 \\
MS & 22.4 \\
MM & 24.7 \\
ML & 28.1 \\
MXL & 30.8 \\
FXS & 20.8 \\
FS & 22.2 \\
FM & 23.6 \\
FL & 25.9 \\
FXL & 26.9 \\
\hline
\end{tabular}

${ }^{a}$ Male (M), Female (F), Extra small (XS), Small (S), Medium (M), Large (L), Extra large (XL). 


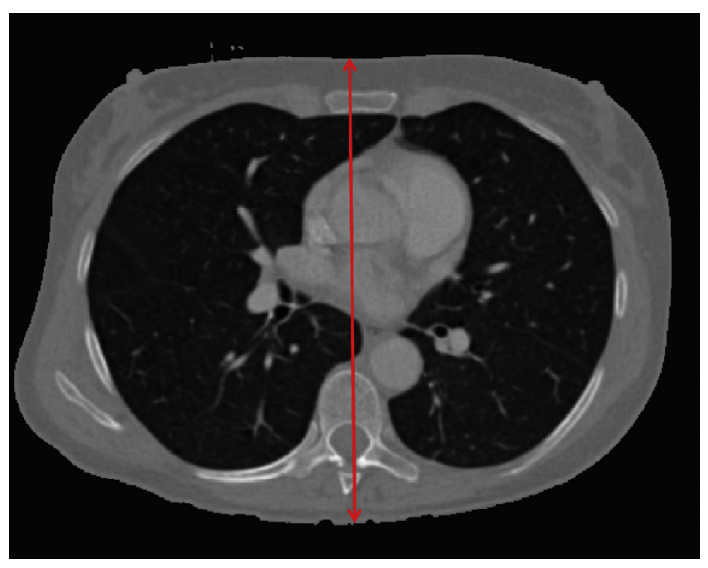

Fig. 1. The arrow indicates the patient thickness measured at the mid-sagittal plane at the mamilla level in anterior-posterior (AP) dimension.

Table 2

$\mathrm{X}$-ray beam, patient and exposure parameters for thorax posterior-anterior (PA) examination in PXCMC simulations.

\begin{tabular}{lll}
\hline Parameter & Reference & Variations \\
\hline Tube voltage $(\mathrm{kV})$ & 120 & $70,80,90,100,110,130^{\mathrm{b}}$ \\
Filtration $(\mathrm{mmAl} / \mathrm{mmCu})$ & $4.0 / 0.2$ & $3.0 / 0.0,5.0 / 0.0,4.0 / 0.1^{\mathrm{b}}$ \\
X-ray system anode & W-anode, & \\
& target angle 20 & \\
Half-value layer $(\mathrm{mmAl})$ & 8.1 & $2.6 ? 8.5^{\mathrm{b}}$ \\
Focus to image distance $(\mathrm{cm})$ & 200 & \\
Focus to skin distance $(\mathrm{cm})$ & 177 & $166.2 ? 177.6^{\mathrm{c}}$ \\
Image width $(\mathrm{cm})$ & $35^{\mathrm{b}}$ & $48^{\mathrm{c}}$ \\
Image height $(\mathrm{cm})$ & 40 & \\
X-ray beam width $(\mathrm{cm})$ & 31.0 & $39.9 ? 42.6^{\mathrm{c}}$ \\
X-ray beam height $(\mathrm{cm})$ & 35.4 & $33.2 ? 35.5^{\mathrm{c}}$ \\
$Z_{\text {ref }}{ }^{\mathrm{a}}$ & 55 & \\
Phantom exit-image distance & 3.0 & \\
Phantom height $(\mathrm{cm})$ & 178.6 & $68.6 ? 174.1^{\mathrm{c}}$ \\
Phantom mass $(\mathrm{kg})$ & 73.2 & $19.4 ? 30.8^{\mathrm{c}}$ \\
Phantom trunk thickness $(\mathrm{cm})$ & 20.0 &
\end{tabular}

a $Z_{\text {ref }}$ is the $z$ coordinate of an arbitrary point inside the phantom, through which the central axis of the X-ray beam is directed.

b Radiation quality: image width is adjusted for the PCXMC phantom with standard dimensions. Variations for tube voltage, filtration and HVL were applied.

c Patient thickness: image width for all patients is adjusted based on the largest thickness-adjusted PCXMC phantom. Variations for FSD, X-ray beam width and height, phantom mass and phantom trunk thickness were applied.

The PCXMC 2.0 [12] includes mathematical hermaphrodite phantoms of ages $0,1,5,10,15$ and adult. In this study, PCXMC was used to determine the effect of radiation quality and patient thickness on conversion factors. The standard dimensions for an adult phantom in PCXMC (height, mass and trunk thickness) are given in the Reference column in Table 2. The X-ray spectra (see Table 2) used in PCXMC simulations was generated in PCXMC. An incident air kerma (input parameter in PCXMC) of $1 \mathrm{mGy}$ was used for the simulations and organ dose conversion factors were calculated relative to the incident air kerma.

Simulations for different patient sizes were performed in PCXMC by using the phantom masses that result in the measured male patient thicknesses (anterior-posterior thicknesses of 19.4$30.8 \mathrm{~cm}$ ). Phantom masses were calculated based on the standard height of the PCXMC phantom (see Table 2) and the measured patient thicknesses (see Table 1). The image width was adjusted so that the X-ray beam covered the lungs of the largest patient (MXL), and this image width was used for all other male patient sizes (see Tables 1 and 2). PCXMC scales the patient model and the X-ray beam dimensions based on the given height and mass of the phantom. The effect of radiation quality on the conversion factors was determined in PCXMC with standard dimensions with four different filtration combinations together with a range of tube voltages (Table 2). Conversion factors were presented as a function of the tube voltage and the half-value layer (HVL).

Lungs and breasts were segmented from the patient CT images by using 3DSlicer (version 4.4) and Matlab (version R2014b), and organ doses were determined from these volumes of interest (VOIs). Breasts were segmented only for the female patients (anterior-posterior thicknesses of $20.8-26.9 \mathrm{~cm}$ ). In PCXMC, the absorbed doses to lungs and breasts were determined as organ doses in the hermaphrodite phantom. Dose conversion factors were calculated by dividing the organ doses from ImpactMC and PCXMC simulations by the incident air kerma and expressed in $\mu \mathrm{Gy} / 100 \mu \mathrm{Gy}$. This unit was selected in order to represent the conversion factors as percentages, when the incident air kerma is $100 \mu \mathrm{Gy}$. The value of $100 \mu \mathrm{Gy}$ is close to the incident air kerma calculated based on the Finnish DRL as the entrance surface air kerma for thorax PA examination $(0.12 \mathrm{mGy})$ [28] and the backscatter factor of 1.4 [27]. The reference conversion factors were calculated for the PCXMC phantom with standard dimensions. The variation ranges in the conversion factors were found at the minimum and maximum values of the half-value layer (HVL) and the anterior-posterior (AP) thickness. The relative variation ranges of the conversion factors were calculated as the difference between the maximum and minimum values relative to the mean value and the reference value.

\section{Results}

\subsection{Radiation quality}

In Figs. 3a-b and 4a-b, conversion factors from incident air kerma to lung and breast doses, respectively, for the thorax PA examination simulated in PCXMC with different radiation qualities are presented as a function of the tube voltage and HVL. The tube voltage is not a good parameter for specifying the radiation quality alone; the total filtration also has to be defined. As a function of HVL, the conversion factors are more convergent than as a function of the tube voltage.

\subsection{Patient thickness}

In Fig. 5, the organ dose conversion factors from ImpactMC simulations for patients with different anterior-posterior thicknesses (Table 1) are compared with the values calculated using the thickness-adjusted mathematical phantoms in PCXMC.

When the X-rays penetrate through the body, the effect of patient thickness can be seen, as the dose inside the patient decreases as a function of the patient thickness (Fig. 5). Linear regression lines were fitted to the ImpactMC results for lungs and breasts, and the corresponding squared linear correlation coefficients $\left(R^{2}\right)$ are 0.93 and 0.97 , respectively. The ImpactMC and PCXMC results for lungs have the same inclination, the ImpactMC results being up to $16 \%$ lower than the PCXMC results at the same patient thickness. The ImpactMC results for breasts show a different inclination than the PCXMC results, and for small patients, the ImpactMC results are up to $52 \%$ higher than the PCXMC results, and for large patients, the ImpactMC results are up to 55\% lower than the PCXMC results of patients at the same patient thickness: $208 \mathrm{~mm}$ (female XS) for small patients and $269 \mathrm{~mm}$ (female XL) for large patients, respectively.

Table 3 presents the conversion factors according to Fig. 5 and the difference between the maximum and minimum doses relative to the mean dose and the reference dose. Reference values are 

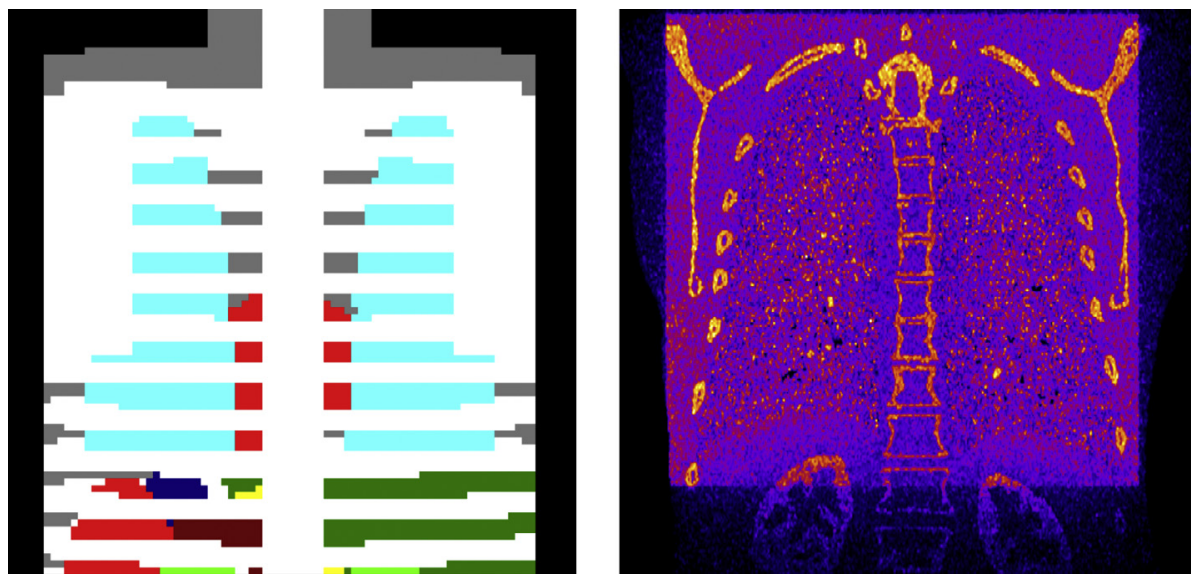

Fig. 2. Examples of beam eye views in thorax PA projection in PCXMC simulations (left) and in ImpactMC simulations (right). In the left image, different colours represent organs and the white areas are bones. In the right image, yellow/lightest is the highest dose and blue/darkest is the lowest. (For interpretation of the references to colour in this figure legend, the reader is referred to the web version of this article.)

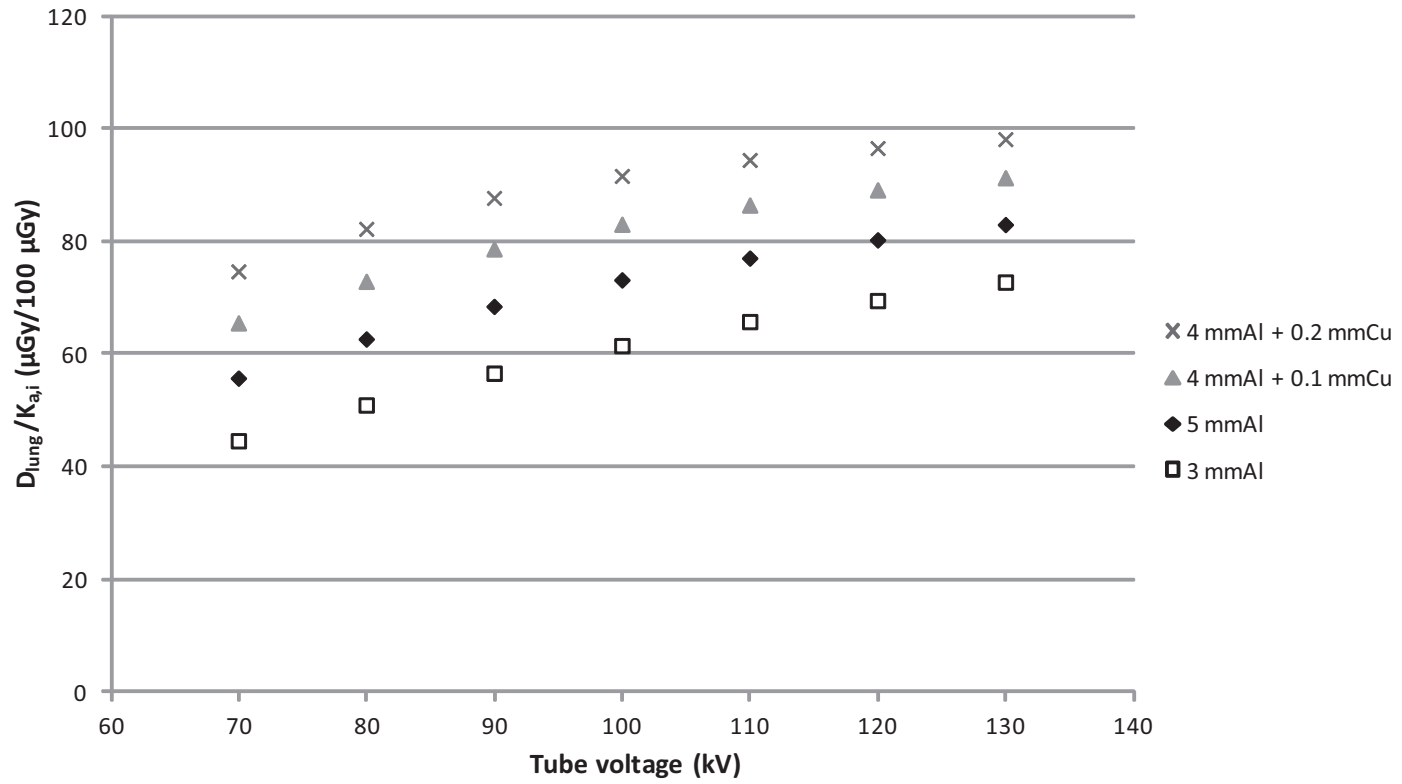

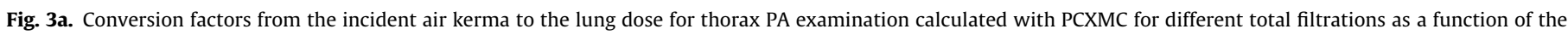
tube voltage.

calculated for the PCXMC phantom with standard dimensions (Table 2). Table 3 represents the variations that may be encountered in lung and breast doses, even though the measurable incident dose level is the same. The highest variations relative to the mean and reference values were found in ImpactMC results for breasts. According to the PCXMC results, the organ doses differ by a factor of up to 3.5 depending on the tube voltage and filtration or HVL and filtration combinations. Patient thickness has an effect of slightly larger order of magnitude; for a small-sized adult, organ doses are up to 5.7 times larger than for a large adult. Compared to the reference values, the relative variation range in organ dose conversion factors is up to $74 \%$ for different radiation qualities and $122 \%$ for different patient thicknesses.

\subsection{Uncertainty calculation}

In Monte Carlo simulations, uncertainties include statistical uncertainties and uncertainties related to the repeatability of the dose determination, but the major source of uncertainties are the uncertainties related to the attenuation coefficients and inadequacies in the description of the X-ray source and the patient. The number of simulated interactions in the organ considered defines the level statistical uncertainties [12].

In ImpactMC, $10^{9}$ photons were used, and the statistical uncertainty was estimated to be $2.0 \%(\mathrm{k}=2)$. The calculated uncertainty based on the minimum and maximum values of the mean doses for the lung and breast VOIs in repeated dose determinations was up to $3.0 \%(\mathrm{k}=2)$. Therefore the total uncertainty of dose values was $3.6 \%(\mathrm{k}=2)$ for the VOIs. In patients, the shapes, volumes and locations of the organs are patient-specific and vary considerably.

In PCXMC, the statistical uncertainties in organ dose values were up to $1.2 \%(\mathrm{k}=2)$ for breasts and up to $0.4 \%(\mathrm{k}=2)$ for lungs at $10^{6}$ photons. The shapes, volumes and locations of the organs in the PCXMC phantom are well defined, but the phantom does not represent a realistic patient. The dominating sources of uncertainties of organ doses in PCXMC are the differences between the phantom and a realistic patient, and the differences between the simulated and true irradiation geometry. 


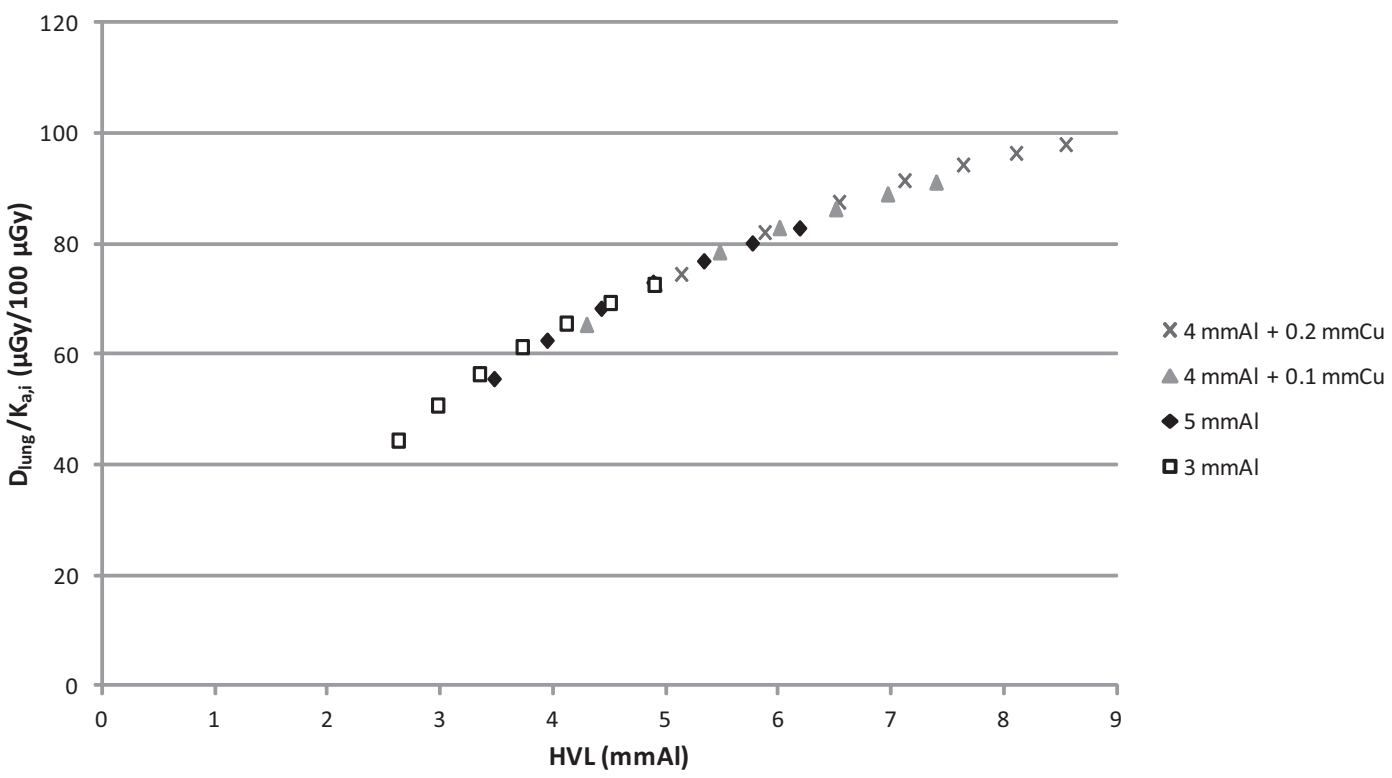

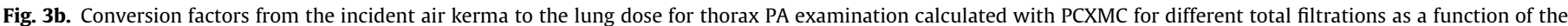
HVL.

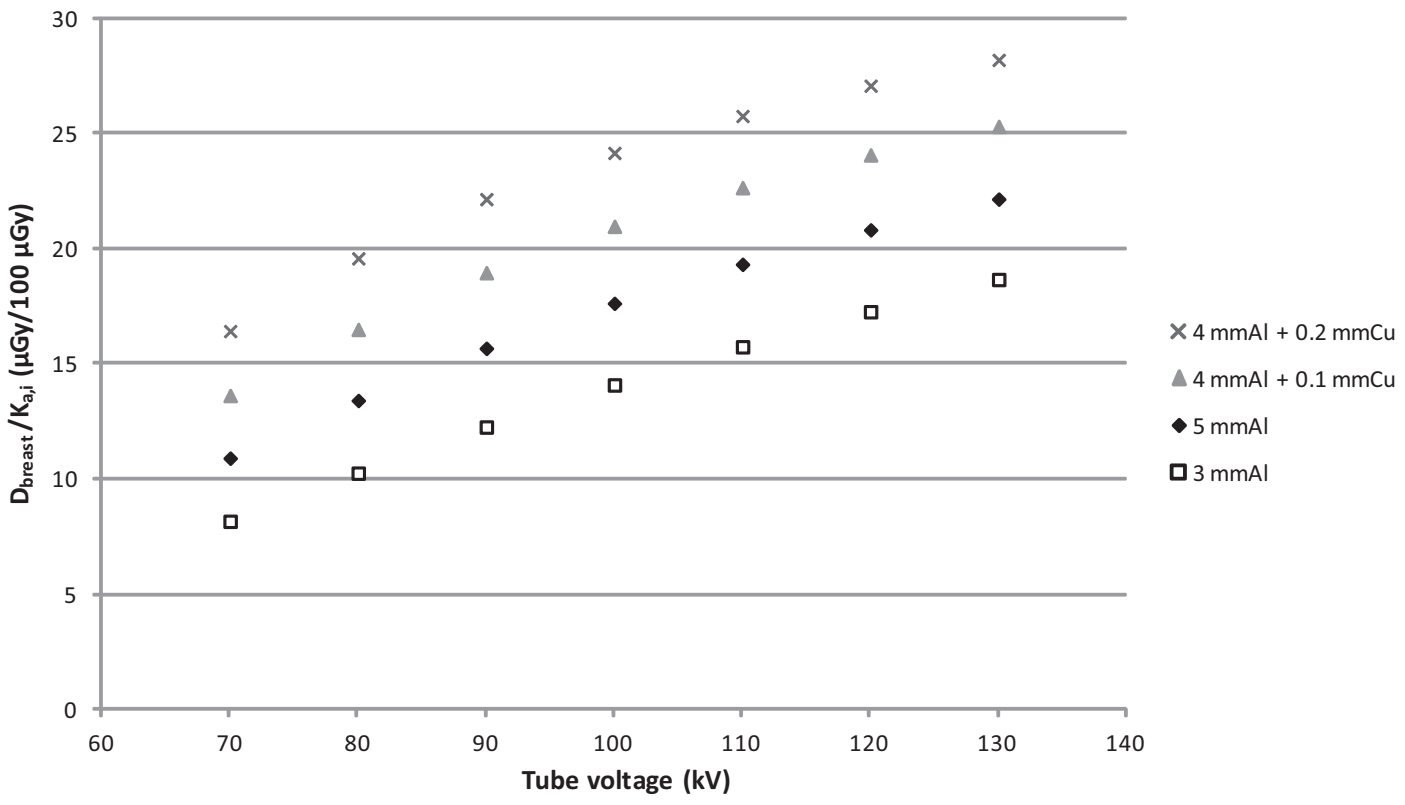

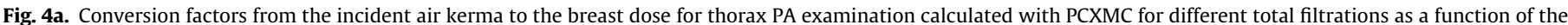
tube voltage.

\section{Discussion}

The international recommendation for the uncertainty of dose measurements in diagnostic radiology is $7 \%(\mathrm{k}=2)$ if the value is used for the optimization of patient doses [21,22,29]. Even a $10 \%$ reduction in the patient dose is a worthwhile objective for optimization [22]. If optimization tasks are close to the $10 \%$ level, this sets high requirements for accuracy in patient dose estimations. This emphasizes the need for correct conversion factors from measurable doses to patient organ doses. The next step, risk estimation, may have large uncertainties of up to an order of magnitude or more [30]. However, an uncertainty of $20 \%$ is acceptable in cases where the organ dose is low and for radiation survey measurements [22], but an uncertainty of $30-50 \%$ can be accepted when organ doses are low [21]. In the present study, uncertainties are associated to the different simulation programs used (ImpactMC and PCXMC) and the dose determination methods. However, large variations in the organ dose conversion factors are related to the choice of a standard-sized patient instead of a specific-sized patient. Moreover, the choice of the radiation quality has a large effect on the organ dose conversion factors.

The highest variation in organ dose conversion factors relative to the reference value, $122 \%$, were found in ImpactMC results for breasts, which is likely to be due to the differences between the size and position of the breasts of the hermaphrodite PCXMC phantom and the female patients. For FS, FM, FL and FXL patients, the breasts were not completely within the primary beam, which is often the clinical situation due to proper lateral and inferior collimation. 


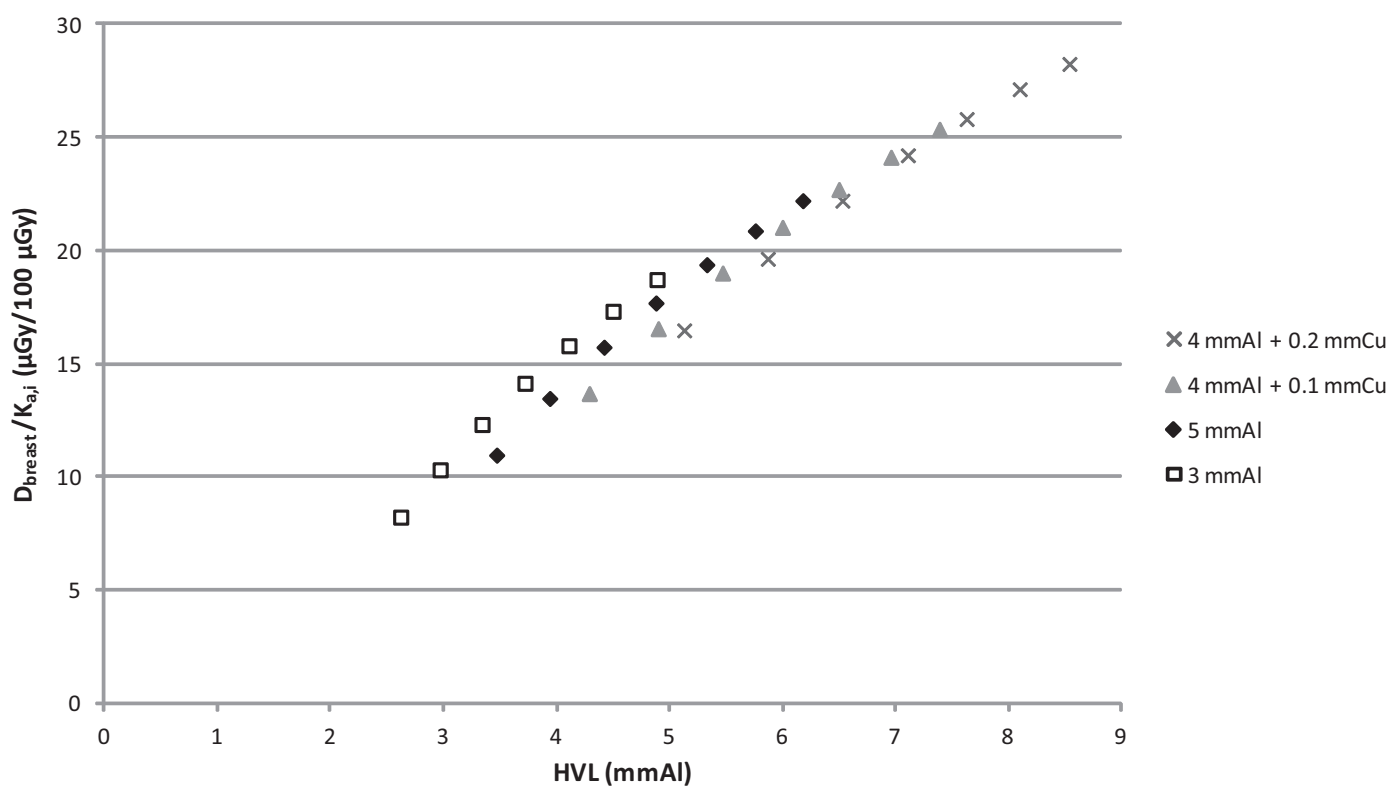

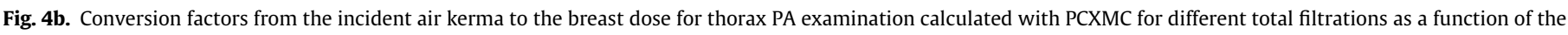
HVL.

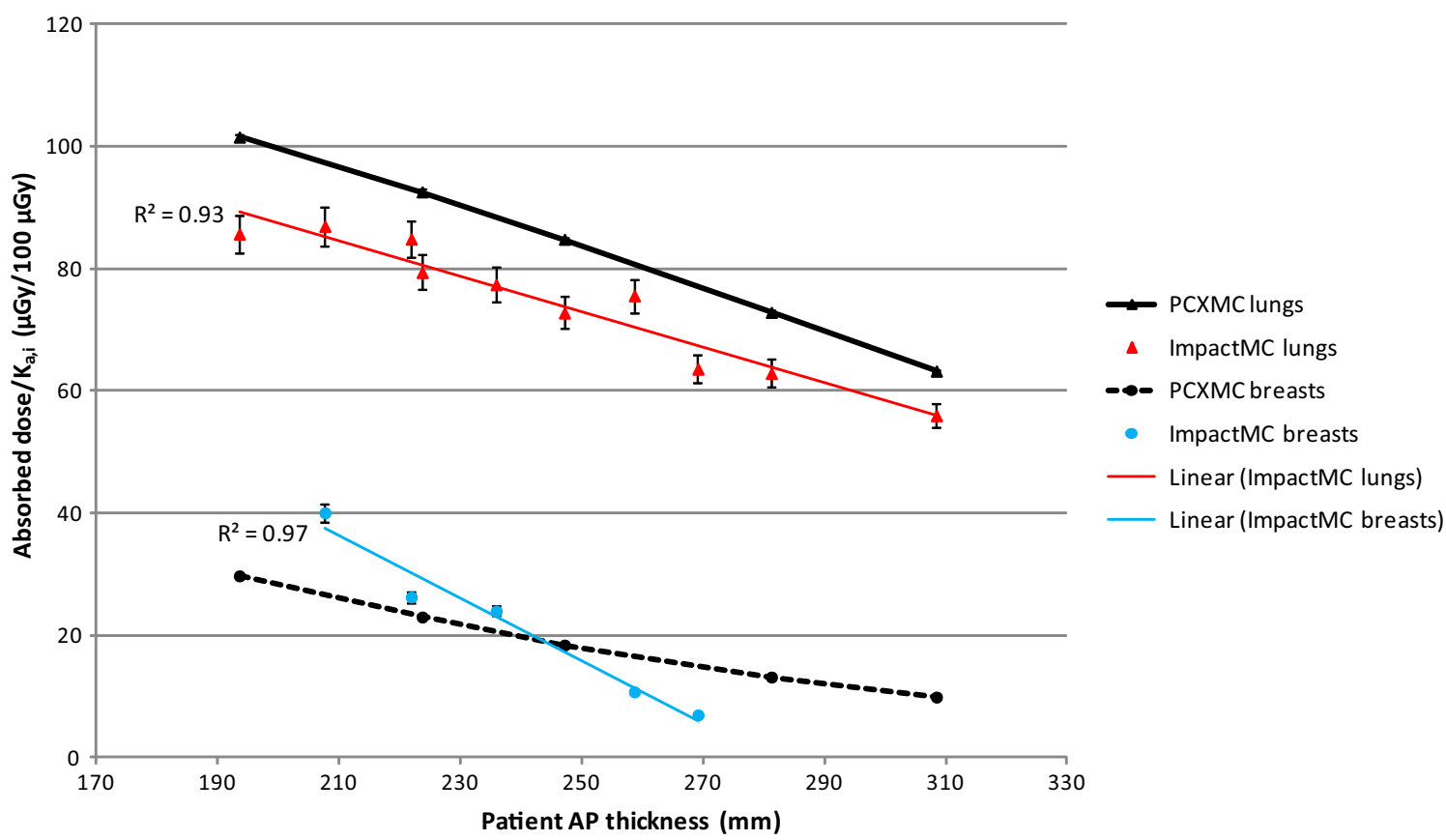

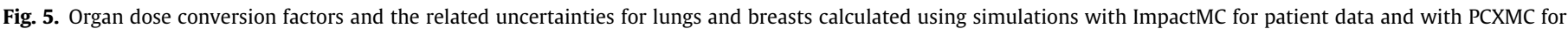
thickness-adjusted phantom data for different patient AP thickness at the reference radiation quality $(120 \mathrm{kV}$ and $4 \mathrm{mmAl}+0.2 \mathrm{mmCu})$.

Therefore, the larger variation ranges in the ImpactMC breast results compared to the PCXMC breast results are due to larger variations in the patient anatomy than in the phantom construction. Moreover, the reference values were calculated for the PCXMC phantom with standard dimensions, which corresponds to a patient size between XS and S (see Tables 1 and 2). For comparison, in the energy range between 60 and $200 \mathrm{keV}$, the range of variation in breast doses between different voxel models have been found to be up to $84 \%$ in the PA projection of whole body irradiations [31].

Both the ImpactMC and PCXMC lung and breast dose conversion factors of the present study showed good linear correlation with the patient AP thickness. The ImpactMC and PCXMC lung results were parallel, but the ImpactMC breast results crossed the PCXMC breast results approximately at the AP thickness of the MM sized patient. These differences might be due to the fact that the thickness adjustment is more realistic for modifying the lung size than the breast size. For medium sized patients, the conversion factors for breasts seem to be similar for patients and mathematical phantoms, at least according to the small patient cohort of the present study. However, as can be seen from the conversion factors for lungs, the thickness-adjusted mathematical phantoms seem to overestimate the lung doses of patients.

In CT examinations of the thorax, dose underestimations of $8-15 \%$ on average depending on patient size with highest 
Table 3

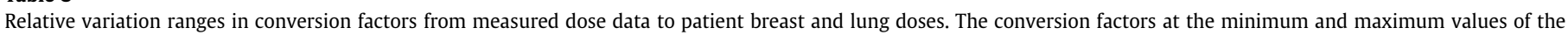
half-value layer (HVL) and the anterior-posterior (AP) thickness are presented.

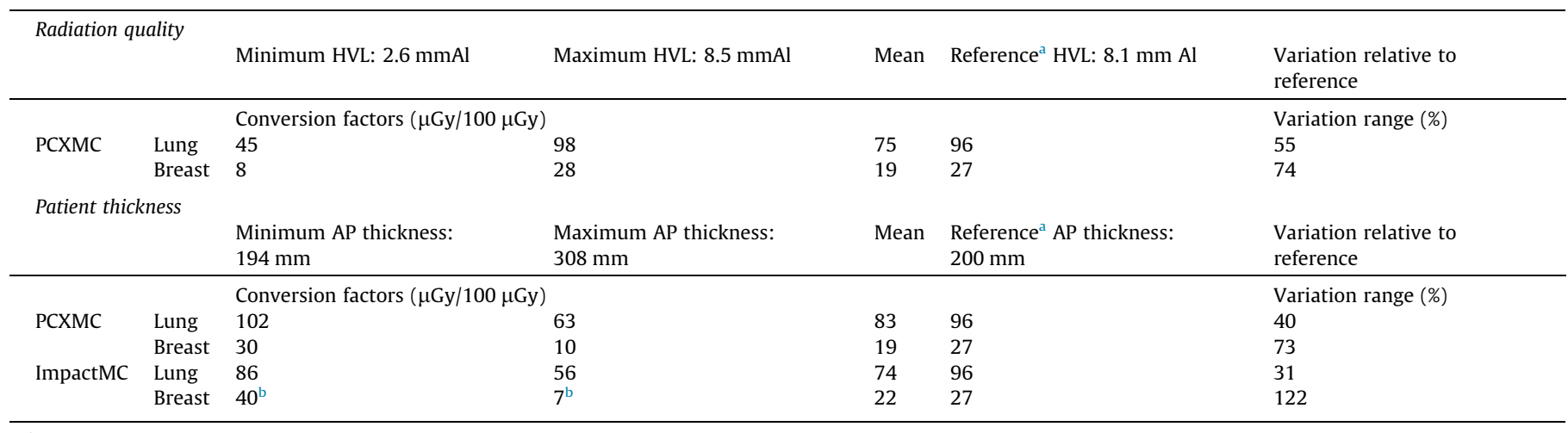

\footnotetext{
a PCXMC phantom with standard dimensions.

b The minimum and maximum AP thicknesses for ImpactMC breast results are $208 \mathrm{~mm}$ and 269 mm, respectively (female extra small and extra large patients).
}

underestimation values of $37 \%$ have been found in Monte Carlo simulations based only on CT data [32]. In projection radiography, however, the internal phantom and patient dose distributions are not as uniform as in CT. It has previously been shown that when patient size is neglected in the choice of the lung dose conversion factor, relative errors of up to $34 \%$ for the PA projection in interventional fluoroscopy procedures can be encountered [33]. Furthermore, the organ dose to an underweight patient will be underestimated and the organ dose to an overweight patient overestimated if patient size is neglected when choosing a dose conversion factor [33]. The variation in conversion factors from entrance surface air kerma to the effective dose between different-sized male and female phantoms has been reported to vary in the range of 1.5-2 in projection radiography [34]. The variation ranges for organ doses can be even higher due, for example, to different $\mathrm{X}$-ray beam positioning [15]. The deviation in lung dose between patients of $100 \mathrm{~kg}$ in mass and $1.7 \mathrm{~m}$ in height and an average adult has been reported to be $27 \%$ in thorax PA examinations, and that the body weight or height alone is a poor parameter for lung dose estimation [35]. It has also been found that a better parameter, the body mass index (BMI), should be used instead, and that the BMI is approximately linearly proportional to the patient trunk thickness [35]. Furthermore, in the energy range between 60 and $200 \mathrm{keV}$, the range of variation in lung doses between different voxel models have been found to be up to $31 \%$ in the PA projection of whole body irradiations [31]. The results of the present study are in line with these previous studies, and further emphasize that the patient thickness has a larger relative effect on the breast dose than on the lung dose due to the position of the breasts at the patient exit in the thorax PA examinations and to the position of the lungs entirely within the primary beam.

The PCXMC results of the present study for different radiation qualities were in a good agreement with published or calculated lung and breast dose conversion factors from the air kerma-area product to organ doses $[7,9,36,37]$. In thorax PA examination, the organ dose in relation to incident air kerma values increased as a function of increasing tube voltage, HVL and total filtration. Filtration of the X-ray spectrum is used to eliminate the low energy photons that would otherwise be absorbed by the patient and thus increase the radiation dose. This dose reduction effect is seen at the patient entrance. In the PA projection, breast and lung dose conversion factors increase as a function of total filtration, because the spectrum has lost the low energy photons and the beam energy has increased when patient's tissues have further filtered medium energy photons. This aspect should be taken into account when entrance surface air kerma values for patients are compared with each other or with the diagnostic reference level. For patients exposed with different filtration, the entrance surface air kerma can be the same, but the absorbed dose in organs can still differ considerably. It has been shown that for low photon energies, the differences in organ doses between different voxel models may amount to hundreds of per cent [31].

The simulation programs include certain study limitations. In PCXMC, the patient model is based on a standard MIRD-type phantom that does not correspond to a realistic patient. In PCXMC, it is possible to scale the phantom size by changing the phantom height and mass. When the actual height and mass of the patient are used to scale the phantom, the resulting phantom thickness does not correspond to the patient thickness [15]. Therefore, the phantom mass has to be adjusted so that resulting the phantom thickness equals the patient thickness. The scaled phantoms used in PCXMC do not represent the anatomical changes of different-sized patients, since in PCXMC the sizes of all organs and tissues are simply increased or decreased according to horizontal and vertical scaling factors. For example, no extra inter-organ fat is inserted. Anatomically, the lung size is not necessarily larger for large patients, but the scaling of the PCXMC phantom also increases the lung size.

Other methodological limitations of this study are related to the patient data available and the image characteristics. The patients were trauma patients and the position of anatomical structures did not represent that of healthy individuals. Moreover, in the supine position, as used for $\mathrm{CT}$, breast tissue has a more lateral location than in the upright position used for thorax PA radiography. Furthermore, inspiration is less pronounced in the supine position, and other organs are also in different positions in upright and supine positions, which may affect the results. Lungs and breasts were segmented from the patient $\mathrm{CT}$ images, which causes variation due to the segmentation method and patient anatomy. The X-ray beam size was the same for all patients simulated in ImpactMC, even though in clinical radiography, the field is always adapted to the individual patient. However, the lungs were fully covered by the X-ray beam, so that the uncertainty related to the use of a standard-sized beam was considered minor. The position of the X-ray detector was estimated to be at the sternum position in order to fix the position similarly for male and female patients, which may cause differences within the patient FDDs.

\section{Conclusions}

The present study aimed to determine the effect of patient thickness on the dose conversion factors by Monte Carlo 
simulations of a thorax PA examination, based on CT examination data of adult male and female patients with different anteriorposterior thicknesses and thickness-adjusted mathematical hermaphrodite phantoms, and the effect of different radiation qualities based on a standard-sized mathematical hermaphrodite phantom. Converting the measurable quantities to patient organ doses is usually based on a standard-sized patient model and a specific radiation quality, which are likely to differ from the real situation. Conversion factors should only be used with comprehensive understanding of the exposure conditions, considering the exposure parameters, exposure geometry and patient anatomy they are valid for. When conversion factors are used for other exposure conditions, the possible large variations in organ doses should be understood. This study demonstrates that patient thickness-specific and radiation quality-specific conversion factors are needed in projection radiography.

\section{Acknowledgements}

Funding: This work was supported by the State Subsidy for University Hospitals in Finland (A.K.).

The authors are grateful to Alexey Sofiev for assistance in image processing.

Previously, information on the methods used for organ dose estimation was collected as part of a Coordinated Research Project (CRP E2.10.08) of the International Atomic Energy Agency.

\section{References}

[1] ICRP (International Commission on Radiological Protection). ICRP Publication 103. The 2007 recommendations of the international commission on radiological protection. Ann ICRP 2007;37(2-3). http://dx.doi.org/10.1016/i. icrp.2007.10.001.

[2] Hall EJ, Brenner DJ. Cancer risks from diagnostic radiology. Br J Radiol 2008;81:362-78. http://dx.doi.org/10.1259/bir/01948454.

[3] Martin CJ. Effective dose: how should it be applied to medical exposures? $\mathrm{Br}$ J Radiol 2007;80(956):639-47. http://dx.doi.org/10.1259/bir/25922439.

[4] AAPM (American Association of Physicists in Medicine). Size-Specific Dose Estimates (SSDE) in Pediatric and Adult Body CT Examinations. Report No. 204. College Park, USA. 2011.

[5] European Commission. European Guidance on Estimating Population Doses from Medical X-Ray Procedures. Radiation Protection No. 154 Luxembourg:EC;2008.

[6] Hart D, Jones D, Wall B. Estimation of effective dose in diagnostic radiology from entrance surface dose and dose-area product measurements. Report NRPB-R262. London:HMSO;1994.

[7] Schultz FW, Geleijns J, Zoetelief J. Calculation of dose conversion factors for posterior-anterior chest radiography of adults with a relatively high-energy Xray spectrum. Br J Radiol 1994;67:775-85. http://dx.doi.org/10.1259/0007$1285-67-800-775$

[8] Le Heron JC. Estimation of effective dose to the patient during medical x-ray examinations from measurements of the dose-area product. Phys Med Biol 1992;37:2117-26. http://dx.doi.org/10.1088/0031-9155/37/11/008.

[9] Petoussi-Henss H, Panzer W, Zankl M, Drexler G. Dose-area product and body doses. Radiat Prot Dosim 1995;57:363-6.

[10] Le Heron J. XDOSE software. Christchurch, New Zealand: National Radiation Laboratory. Ministry of Health; 1994.

[11] Kramer R, Khoury HJ, Vieira JW. CALDose_X-a software tool for the assessment of organ and tissue absorbed doses, effective dose and cancer risks in diagnostic radiology. Phys Med Biol 2008;53:6437-59. http://dx.doi.org 10.1088/0031-9155/53/22/011.

[12] Tapiovaara M, Siiskonen T. PCXMC. A Monte Carlo program for calculating patient doses in medical x-ray examinations. Report STUK-A231. Helsinki:STUK;2008.

[13] Deak P, van Straten M, Shrimpton PC, Zankl M, Kalender WA. Validation of a Monte Carlo tool for patient-specific dose simulations in multi-slice computed tomography. Eur Radiol 2008;18:759-72. http://dx.doi.org/10.1007/s00330007-0815-7.

[14] Schmidt B, Kalender W. A fast voxel-based Monte Carlo method for scannerand patient-specific dose calculations in computed tomography. Phys Medica 2002;18:43-53.
[15] Toroi P, Kelaranta A, Vock P, Siiskonen T, Tapiovaara M, Kosunen A. Interpretation of measured dose data in X-ray imaging. In: 13th international congress of the international radiation protection association. Glasgov, Scotland;2012. Available from: <http://www.irpa.net/members/TS2a. 9.pdf>.

[16] Hart D, Jones D, Wall B. Normalised organ doses for medical X-ray examinations calculated using Monte Carlo techniques. Report NRPB-SR262. Chilton:NRPB;1994.

[17] Helasvuo T. Number of radiological examinations in Finland in 2011. Report STUK-B 161. Helsinki:STUK;2013.

[18] Speets AM, van der Graaf Y, Hoes AW, Kalmijn S, Sachs APE, Rutten MJCM, et al. Chest radiography in general practice: indications, diagnostic yield and consequences for patient management. Br J Gen Pract 2006;56:574-8. http://dx.doi.org/10.1093/fampra/cml027.

[19] Veldkamp WJH, Kroft LJM, Geleijns J. Dose and perceived image quality in chest radiography. Eur J Radiol 2009;72:209-17. http://dx.doi.org/10.1016/ j.ejrad.2009.05.039.

[20] ICRP (International Commission on Radiolgical Protection). ICRP Publication 60. Recommendations of the international commission on radiological protection. Ann ICRP 1990;1991:21.

[21] ICRU (International Commission on Radiation Units and Measurements). Patient dosimetry for X-rays used in medical imaging. ICRU Report 74. J ICRU 2005;5:1-113.

[22] IAEA (International Atomic Energy Agency). Dosimetry in diagnostic radiology: An international code of practice. Technical Report Series No. 457. Vienna:IAEA;2007.

[23] CT Imaging GmbH. User Guide. ImpactMC. Document Version 1.20. Available from: <http://www.ct-imaging.de/images/Downloads/impactmc_usermanual. pdf> (accessed November 2, 2016).

[24] Schmidt B, Saltybaeva N, Kolditz D, Kalender WA. Assessment of patient dose from CT localizer radiographs. Med Phys 2013;40:84301. http://dx.doi.org/ $10.1118 / 1.4813296$.

[25] Schmidt BT, Hupfer M, Saltybaeva N, Kolditz D, Kalender WA Dose optimization for computed tomography localizer radiographs for low-dose lung computed tomography examinations. Invest Radiol 2016;1. http:// dx.doi.org/10.1097/RLI.0000000000000311.

[26] Tapiovaara M, Tapiovaara T. The validation and user's manual of the Spektripaja (2.0) program. Report STUK-TR3. Helsinki:STUK;2008.

[27] European Commission. European Guidelines on Quality Criteria for Diagnostic Radiographic Images. Report EUR 16260. Luxembourg:EC;1996.

[28] STUK (Radiation and Nuclear Safety Authority). Reference levels for the patient's radiation exposure for conventional X-ray examinations of adults. Available from: <http://www.stuk.fi/documents/88234/1106801/decision-93020-2014-26-6-2014-reference-levels-for-the-patients-radiation-exposurefor-conventional-x-ray-examinations-of-adults.pdf/f0b113e1-ba1e-41c2-90771c3229001875> (accessed April 21, 2016).

[29] Wagner LK, Fontenla DP, Kimme-Smith C, Rothenberg LN, Shepard J, Boone JM. Recommendations on performance characteristics of diagnostic exposure meters: report of AAPM diagnostic X-ray imaging task group No. 6. Med Phys 1992;19:231-41. http://dx.doi.org/10.1118/1.596904.

[30] BEIR (Biological Effects of Ionizing Radiation). Health risks from exposure to low levels of ionizing radiation. BEIR VII. Washington (DC): National Academies Press;2006.

[31] Zankl M, Fill U, Petoussi-Henss N, Regulla D. Organ dose conversion coefficients for external photon irradiation of male and female voxel models. Phys Med Biol 2002;47:2367-85. http://dx.doi.org/10.1088/0031-9155/47/14/ 301.

[32] Kalender WA, Saltybaeva N, Kolditz D, Hupfer M, Beister M, Schmidt B. Generating and using patient-specific whole-body models for organ dose estimates in CT with increased accuracy: feasibility and validation. Phys Medica 2014;30:925-33. http://dx.doi.org/10.1016/j.ejmp.2014.09.005.

[33] Johnson P, Lee C, Johnson K, Siragusa D, Bolch WE. The influence of patient size on dose conversion coefficients: a hybrid phantom study for adult cardiac catheterization. Phys Med Biol 2009;54:3613-29. http://dx.doi.org/10.1088/ 0031-9155/54/12/001.

[34] Rannikko S, Ermakov I, Lampinen J, Toivonen M, Karila K, Chervjakov A. Computing patient doses of X-ray examinations using a patient size- and sexadjustable phantom. Br J Radiol 1997;70:708-18.

[35] Tung CJ, Lee CJ, Tsai HY, Tsai SF, Chen IJ. Body size-dependent patient effective dose for diagnostic radiography. Radiat Meas 2008;43:1008-11. http:// dx.doi.org/10.1016/i.radmeas.2007.11.028.

[36] Theocharopoulos N, Perisinakis K, Damilakis J, Varveris H, Gourtsoyiannis N. Comparison of four methods for assessing patient effective dose from radiological examinations. Med Phys 2002;29:2070-9. http://dx.doi.org/ $10.1118 / 1.1500769$

[37] Drexler G, Panzer W, Petoussi N, Zankl M. Effective dose - how effective for patients? Radiat Environ Biophys 1993;32:209-19. 